\title{
A Training Oriented Driving Simulator
}

\author{
Chao Sun, Feng Xie, Xiaocao Feng, Mingmin Zhang, and Zhigeng Pan \\ CAD\&CG State Key Lab. Zhejiang University, Hang Zhou, China, 310027 \\ \{sunchao, xiefeng, fengxiaocao, zmm, zgpan\}@cad.zju.edu.cn
}

\begin{abstract}
In today's China, a growing number of people have the opportunity to have their own cars. This creates tremendous requirement of training of new drivers. At the same time, recent advances in Computer Graphics and Virtual Reality system have brought the new opportunities for the development of driving simulation. This paper describes a driving simulator named TODS which is the acronym of training oriented driving simulator, developed for rigorous driving training and for Computer Graphics study. TODS is designed under the human-in-the-loop real-time simulation mode providing $120^{\circ}$ horizontal FOV(Field of View) and realistic visual feedback to give the driver a realistic feeling of immersion similar to the feeling in a real moving vehicle. TODS utilizes state-of-the-art real-time rendering techniques in Vertex Shader and Pixel Shader [1] to make it more flexible to implement more real-time rendering algorithms in GPU (Graphic Processing Unit) so as to enhance the system function. TODS's scene model is designed and constructed according to the "Driving Test Specification" of China to meet the demands of rigorous driving training. And a kind of modular construction method is used in the work of scene authoring in TODS.
\end{abstract}

Keywords: Driving simulation, Real-Time Simulation, Real-Time Rendering, Computer Graphics, GPU, Shader, Dynamic Texture Mapping.

\section{Introduction}

A driving simulator is a virtual reality tool that supports a variety of training, research, and design applications. It has advantages over comparable vehicle testing in many aspects including economy, safety, reproducibility, convenience of changing vehicle models and ease of data acquisition. In China, more and more individuals have the opportunity to have a car in recent years. This increases the burden of the training school for drivers. On the other hand, recent advances in Computer Graphics and Virtual Reality system are leading to the new opportunities for the development of driving simulation. To meet the increasing demand of driving training, we develop a training oriented driving simulator named TODS which is the acronym of training oriented driving simulator, based on state-of-the-art CG and VR science and technology. In addition to the common features of general driving simulators, we also implement several distinct functions in our study -- $120^{\circ}$ horizontal field of view, high-resolution visual system to provide realistic visual feedback, more authentic effect of mirror in the virtual environment, precise appearance and size of scene model according to the "Driving Test Specification" for rigorous training. 
In the following sections, this paper describes TODS from four aspects: real-time simulation system, visual system, system enhancement based on programmable GPU and scene authoring. In the final part of this paper, we make several conclusions.

\section{Related Work}

Driving simulation research was first conducted in aircraft simulation. The pioneering work in human-in-the-loop driving simulation [2, 3] in the 1970s by General Motors $®$ and Virginia Polytechnic Institute and State University lasted for three decades and made great advances in this area of study. Our study is also based on the human-in-the-loop mode. We will discuss it in detail in next section.

In 1990s, several outstanding simulators were built. The Center for Computer Aided Design at the University of IOWA developed the IOWA Driving Simulator [4, 5]. It includes a large hexapod motion base and operational gauges and instruments. The driver receives haptic feedback through the steering wheel, accelerator, and brake pedal. Within the vehicle cab, high-resolution, textured graphics generated by an Evans \& Sutherland ESIG 2000 are projected onto a screen in front of the cab and a smaller screen behind the cab visible through real rear-view mirrors. There is still further work that can be done to create more realistic visual feedback than IOWA with the help of the latest Computer Graphics science and technology. We propose our methods in the following sections of "visual system" and "system enhancement based on GPU" in this paper.

The rapidly increasing power of computers makes it possible to produce cheaper driving simulators. For example, Systems Technology Incorporated ${ }^{\circledR}\left(\right.$ STI $\left.{ }^{\circ}\right)$ has developed a desktop configuration that uses personal computers for graphics and vehicle dynamics $[6,7]$. They have done innovative work in this field. However, the visual fidelity is not very high in these simulators. Narrow FOV (Field of View) becomes a crucial limitation. This has been changed in our study. And it will be described in detail in the section of "visual system" in this paper.

\section{Real-Time Simulation System}

The essence of a driving simulation has two aspects. One is the simulator's feedback that a driver receives responding to his/her input commands, another is his/her subsequent control responses induced by that feedback. Therefore, the realness of a simulation mainly depends on the fidelity feedback of visual image displayed on the screens generated by the Visual System in real time and the correct new state of the whole scene including your own car, traffic aids, autonomous cars etc. The new state is generated by the Physics and AI module. According to this, we utilize a nested-control-loop real-time simulation system developed from human-in-the-loop mode $[2,3]$ in TODS.

Figure 1 illustrates a basic concept of TODS's real-time simulation system. As can be seen in the block diagram, there are 3 layers of loop in the system - Operation Loop, Rendering Loop and Physics Loop. 
1. In the Operation Loop, driver inputs his/her control operations to the inner loops through the simulator controls - steering wheel, break, throttle, clutch, etc. and get the feedback through the visual system feedback. The period of Operation Loop $\triangle \mathrm{T}$ mainly depends on the driver's response speed.

2. In the Rendering Loop, GPU renders the 3-D scene to the Frame Buffer through its fixed pipeline for some normal purpose. And we also employ Vertex Shader and Pixel Shader to implement more advanced effects, such as dynamic texture mapping for some special purpose. We will give a detailed explanation in the following section. The period of Rendering Loop - $d t^{\prime}$ of a typical scene in TODS is about $30 \mathrm{~ms}$ on our system. That means the FPS of our system is about 33 .

3. In the Physics Loop, the Physics module calculates the driving car's position, velocity, acceleration, and orientation. In the scenes which have autonomous cars and traffic aids, the AI module is in charge of those cars' behavior. The period of Physics Loop $-d t$ is $10 \mathrm{~ms}$, which is set by us. It's the shortest period among the three, because in every single period of Physics Loop the physical event is computed on CPU by linear differential equations, the shorter the time the more accurate the results.

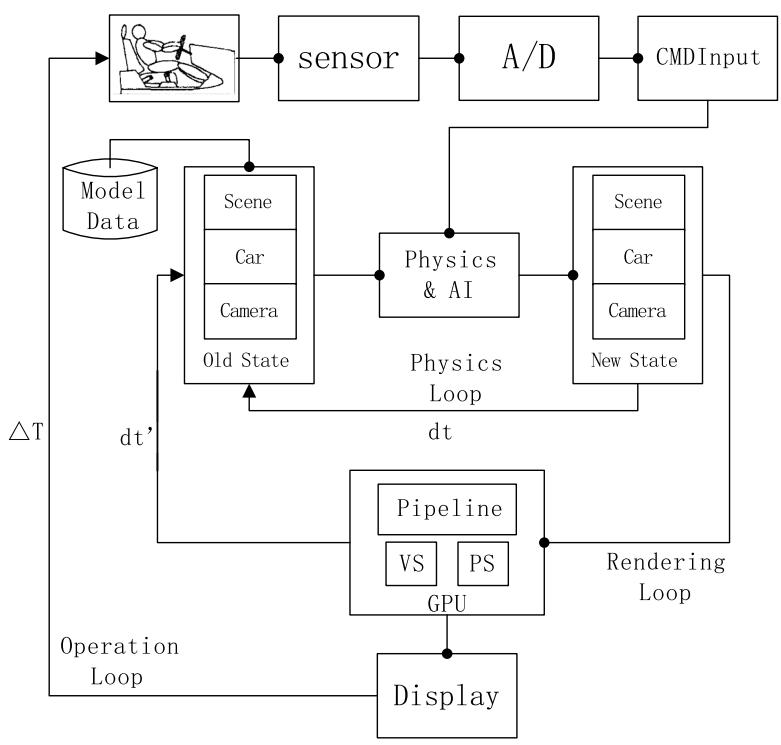

Fig. 1. Real-time simulation system of TODS is a human-in-the-loop mode. Human is in the outer loop operating the control equipments. GPU and CPU are in the inner loops engaged in the work of rendering, physical simulation and AI processing. In this diagram, triangle-arrows indicate control flow and point-arrows indicate data flow.

\section{Visual System}

For a driving simulator, it is most important to provide a realistic feeling of immersion similar to the feeling in a real moving vehicle. During driving, drivers' visual impression 
is the most significant cue for him/her to control the vehicle. Therefore, providing a realistic visual feel is the challenge to the visual system of a driving simulator. Since on the driver's seat of a real car, the front, left and right side are almost all in the driver's vision scope, providing a $120^{\circ}$ horizontal field of view with high resolution graphics in the visual system is essential for the driver to have a realistic driving feel and react to the driving environment precisely.

In our study, Matrox ${ }^{\circledR}$ TripleHead2Go ${ }^{\mathrm{TM}}$ provides $3072 \times 768$ high-resolution images on three monitors which are arranged according to the form of an arc, and the specific frustums are designed to project precise images on each of the screens so that the images can be assembled seamlessly.

Figure 2 (a) shows the form of arranging the monitors and a typical driving scene of TODS. Figure 2 (b) illustrates how to set up the frustums that determine the projection matrixes.

At first, the angle of two contiguous screens - $\alpha$, and the distance from driver's eye to screen $-d$, is preset. And then all of the parameters of these 3 frustums can be obtained by the following equations.

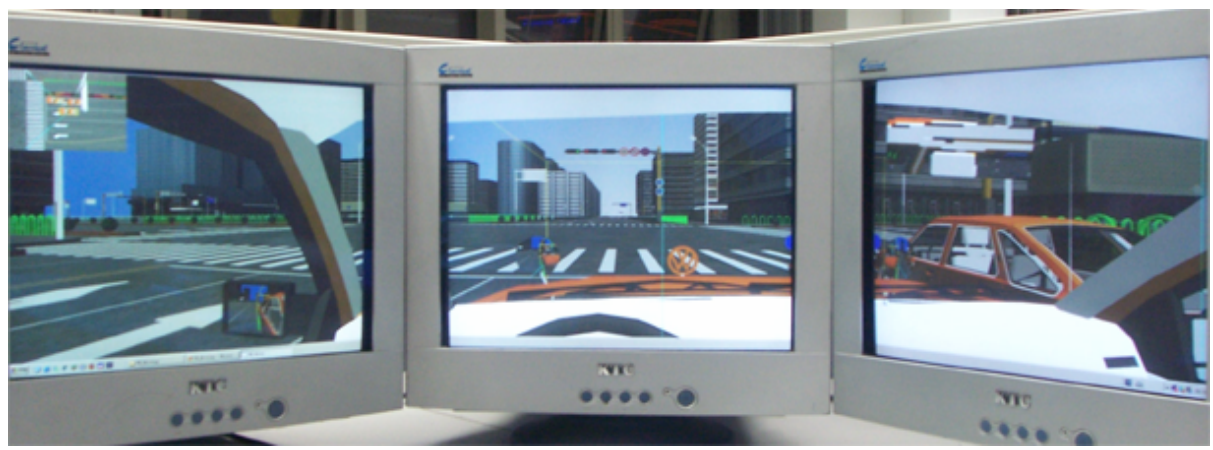

(a)

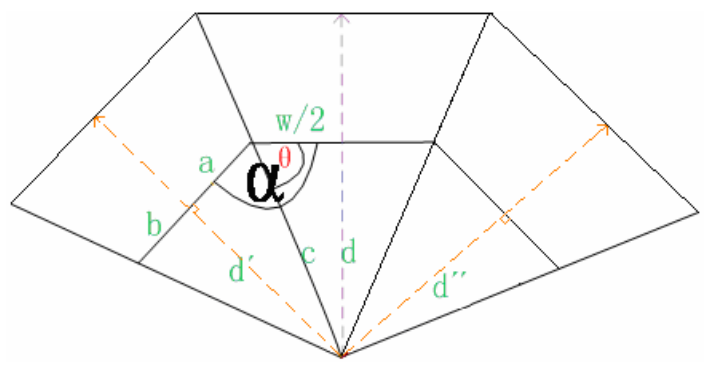

(b)

Fig. 2. A kind of display equipment and a typical running scene of TODS are shown in (a). Different display equipments e.g. projectors can be used for different occasions. The angle of two contiguous screens is preset, so as to provide a $120^{\circ}$ horizontal FOV by 3 screens. The frustums' configuration is shown in (b). $W$ is the width of a screen. 


$$
\begin{gathered}
c=\sqrt{(w / 2)^{2}+d^{2}}, \theta=\arctan (2 d / w) \\
d^{\prime}=\sin (\alpha-\theta) \times c \\
a=\cos (\alpha-\theta) \times c, b=w-a
\end{gathered}
$$

Note that, the left and right frustums can be asymmetric projection. In other words, $a$ is unnecessarily equal to $b$.

\section{System Enhancement Based on GPU}

In our study, some real-time rendering techniques based on programmable Vertex Shader and Pixel Shader are utilized to make it more flexible to implement more real-time rendering algorithms in the GPU and relieve the burden of CPU at the same time. As we know, the rear-view mirror is an important tool for drivers to understand the situation of the rear and sides of his/her car. Therefore providing authentic effect of mirror in the virtual environment is essential for a driving simulator.

There are two kinds of rear-view mirrors on a real training car, planar mirror and convex mirror. Figure 3 presents the views in these two kinds of mirrors on a real training car.
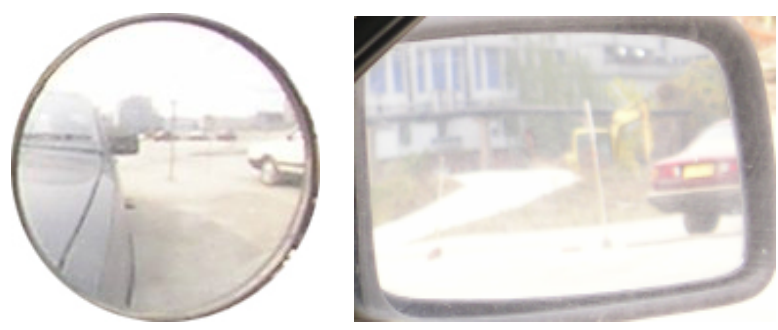

Fig. 3. The views of these two kinds of mirrors on a real car are important for drivers

To simulate these rear-view mirrors, the method proposed by IOWA is to project rear scene of the car onto another small screen behind the cab which is visible through real mirrors $[4,5]$. In our study, Dynamic Texture Mapping $[8,9,10]$ technique based on the VS and PS is employed to solve this problem.

First, the whole scene is rendered to a Target Texture instead of Frame Buffer. We can regard it as an image on the near plane of a specific frustum. This specific frustum is mirror symmetrical with the frustum which is created as an imaginary driver's eyes looking at the virtual mirror. Second, the texture coordinates of the vertex $A$ and $B$ is calculated by Vertex Shader by projecting them onto the near plane. Third, in Pixel Shader, the correct image is mapped on the mirror in accordance with the texture coordinates acquired from previous step. Figure 4 illustrates a function diagram of this method in our study. $A B$ is the mirror plane; $C$ is the imaginary driver's eyes' position. $C$ ' and $C$ are symmetrical with respect to the mirror plane. The whole scene is projected 


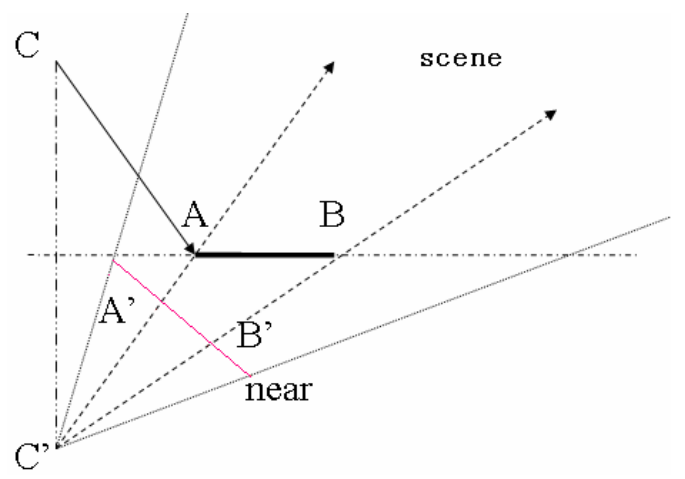

Fig. 4. The function diagram illustrates how to simulate the effect of training cars' planar rear-view mirrors in TODS

on the near plane and $A^{\prime} B^{\prime}$ is the projection of $A B$. The dynamic texture is on the near plane and the texture coordinates of a point on $A B$ is just the point's projection on $A^{\prime} B^{\prime}$.

For a convex mirror, the first step is the same as that of the planar mirror. However, the second and third step is different. Since the convex reflect more landscape, the $A^{\prime} B^{\prime}$ has greater scope than that of a planar mirror. Figure 5 shows the function diagram of the method through which we simulate a convex mirror. In the second step, after the point $A$ and $B$ have been projected to $A^{\prime}$ ' and $B^{\prime}$ on the near plane, $A^{\prime}$ ' and $B^{\prime}$ will extend to $A$ " ' and $B$ "' so as to involve more pixels in $A$ ' ' $B$ " ' to approximate the visible scope of a real convex mirror, and now, $A$ ' ' and $B$ '" are the new texture coordinates of $A$ and $B$. The stretch length- $A A^{\prime}$ " or $B^{\prime} B^{\prime \prime}$ is a projection of $A A^{\prime \prime}$ or $B B^{\prime}$.' Note, the stretch length acquired by means of such projection is not strict. However, it becomes possible not only to reduce computing cost but also to provide sufficient accuracy in our experiment. All these treatments are performed in Vertex Shader. In the third step, before the image in the scope of $A$ ' ' $B$ ' ' is mapped on the mirror mesh, a normal map is utilized to modify the texture coordinates of each pixel (fragment) on the mirror so as to approximate the distortion effect on a real convex. All treatments in the third step are performed in Pixel Shader.

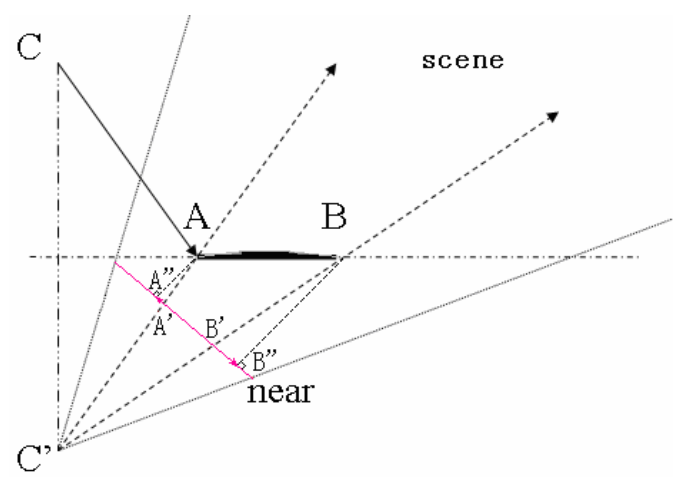

Fig. 5. The function diagram illustrates how to simulate the effect of convex rear-view mirrors on a training car in TODS 

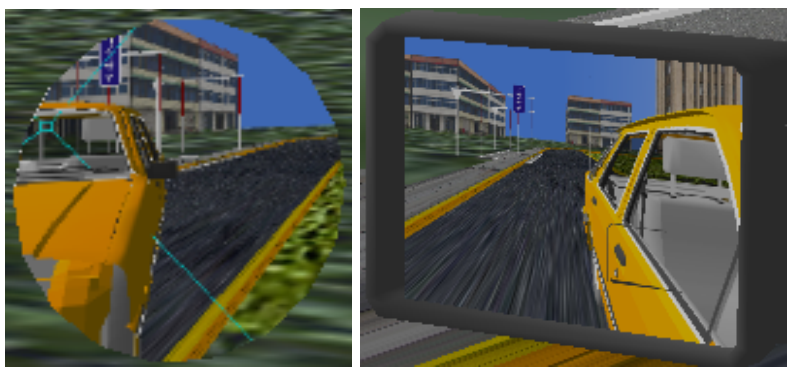

Fig. 6. The simulation of these two kinds of mirror in a virtual environment of TODS are shown in this figure

Figure 6 shows snapshots of two mirrors generated by TODS when the application is running.

\section{Scene Authoring}

Driving simulation requires scene databases of large geographic regions with properly constructed roads, highway, terrain, traffic aids, and appropriate culture features. Aside from those, as a training oriented driving simulator, TODS also focuses on the virtual practice scenes which aim at specialized driving exam items such as "Discus Road", "One Side Bridge", "Quarter Turn" and so on. As can be seen in figure 7, each leaf node of this dendrogram is a single scene of TODS. On the side of "Exam Practice", the appearance and size of every scene model is designed and constructed according to the "Driving Test Specification" of China. Figure 8 shows several scenes of Exam Practice in TODS.

On the other hand, the scenes of "Road Practice", including "City Road", "Mountain Road" and "Highway" are much larger than the scenes of "Exam Practice". At the same

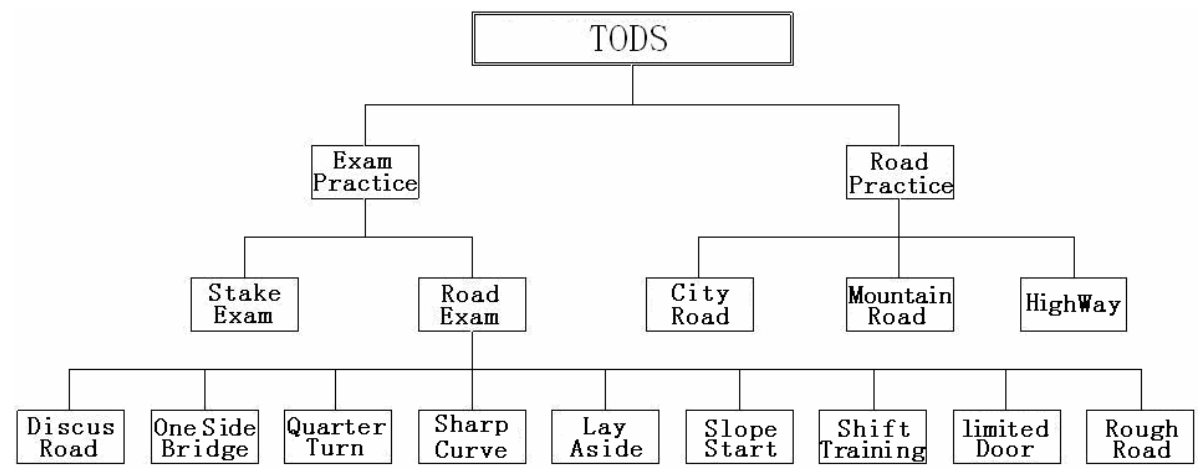

Fig. 7. Dendrogram of scenes in TODS 

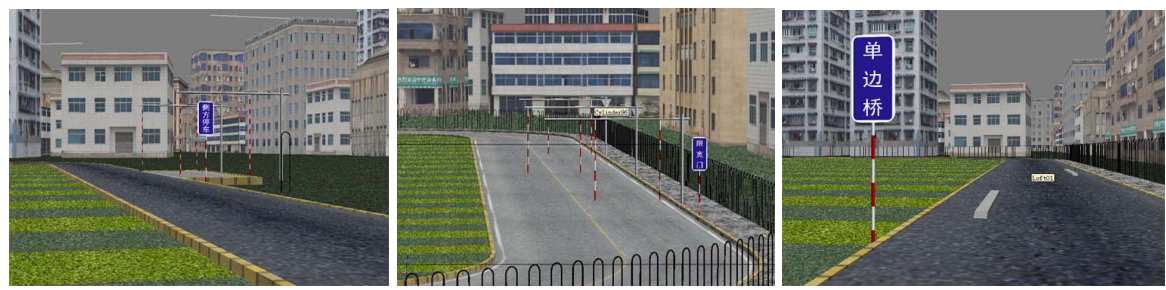

Fig. 8. Several scenes for Exam Practice in TODS, from left to right they are "Lay Aside", "Limited Door", and "One Side Bridge". They are designed and constructed according to the "Driving Test Specification" of China.

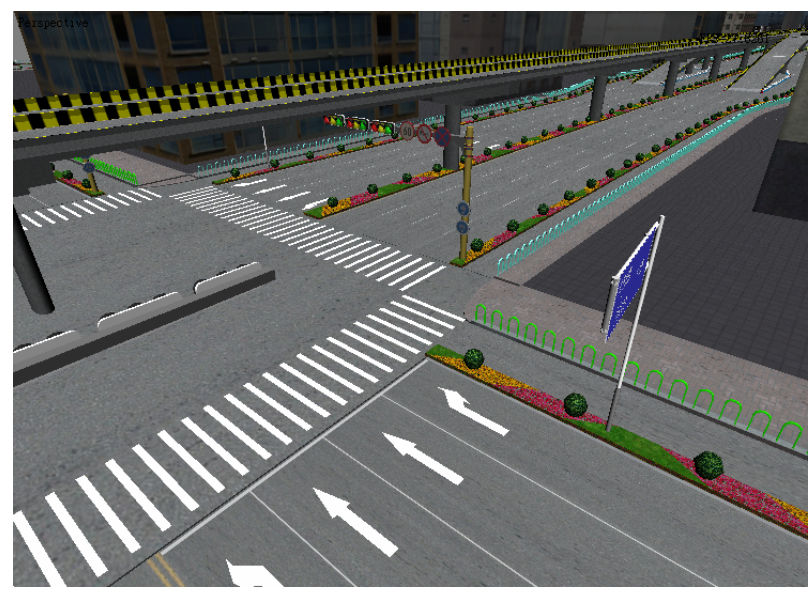

(a)

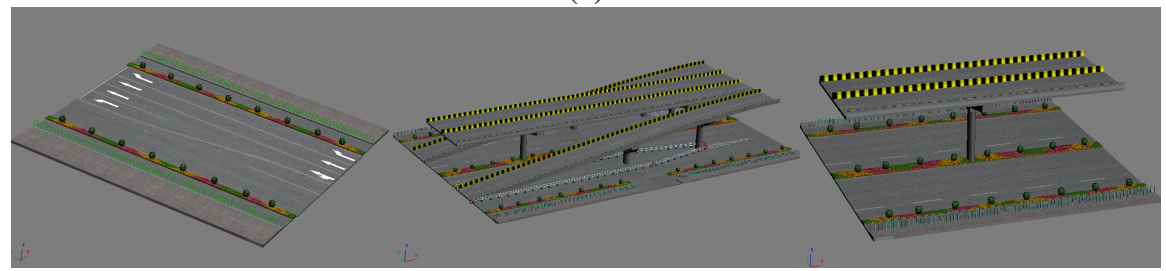

(b)

Fig. 9. The scene of "City Road" as shown in (a) is constructed by several units as shown in (b)

time, there exists no "specification" to restrict how to model these scenes. Therefore, we utilize a kind of modular construction method to design and construct the large scene with standardized units and dimensions. And this method has two purposes:

(1) By dividing a big scene into small units, we can give each of these units a bounding volume, and then we can only render the units whose bounding volume is within the view frustum. This increases the rendering efficiency. (2) If a big scene is designed with standardized units and dimensions, then we can "arrange and fix" this scene more easily. This increases the modeling flexibility.

Figure 9 demonstrates several units and a typical scene of "City Road". 


\section{Conclusions}

A driving simulator named TODS based on state-of-the-art Computer Graphics and VR system technology has been developed.

TODS is designed under the human-in-the-loop real-time simulation system mode. Comparing to common driving simulators, it provides $120^{\circ}$ horizontal FOV and realistic visual feedback to give the driver a realistic feeling of immersion similar to the feeling in a real moving vehicle. TODS utilizes state-of-the-art real-time rendering techniques in Vertex Shader and Pixel Shader to make it more flexible to implement more real-time rendering algorithms in GPU so as to enhance the system function. TODS's scene model is designed and constructed according to the "Driving Test Specification" of China to meet the demands of rigorous driving training. And a kind of modular construction method is used in the work of scene authoring in TODS.

There are still many visual and physical factors which have important influence on driving, e.g. weather, high light reflex, water on the front window, strong wind, should be simulated. Simulation of these kinds of phenomenon effecting on driving is the future research work of TODS.

TODS will be used for rigorous driving training and for Computer Graphics study. More research and study work on latest CG science and technology based on TODS are underway.

\section{References}

1. Fernando, R., Kilgard, M.J.: The Cg Tutorial. The Definitive Guide to Programmable Real-Time Graphics. Addison Wesley, Reading (2003)

2. Wierwille, W.W.: Driving Simulator Design for Realistic Handling. In: Sachs, H.K., Swets, Zeitlinger. (eds.) Proceedings of the Third International Conference on Vehicle Systems Dynamics, B.V., Amsterdam (1975)

3. VPI-SU: (Accessed 2007.04), web site http://hci.ise.vt.edu/ vasl

4. Cremer, J., Kearney, J., Papelis, Y.: Driving Simulation: Challenges for VR Technology. IEEE Computer Graphics and Applications, 16-20 (September 1996)

5. Center for Computer Aided Design: (Accessed 2007), web site http://www.ccad.uiowa.edu/

6. Allen, R.W., Rosenthal, T.J., et al.: A Vehicle Dynamics Model for Low Cost. In: PC Based Driving Simulations Conference Proceedings DSC'97, pp. 153-164 (September 1997)

7. Systems Technology Incorporated: (Accessed 2007), web site http://www.systemstech.com/

8. Fernando, R., Kilgard, M.J.: The Cg Tutorial. The Definitive Guide to Programmable Real-Time Graphics. ch. 7: Environment Mapping. Addison Wesley, Reading (2003)

9. Ramamoorthi, R., Hanrahan, P.: An Efficient Representation for Irradiance Environment maps. In: Proceeding of SIGGRAPH 2001 (2001)

10. Bjorke, K.: Image-Based Lighting. In: GPU Gems: Programming Techniques, Tips, and Tricks for Real-Time Graphics, ch. 19. Addison Wesley Press, Reading (2004) 